\title{
Sentimenti mescolati
}

Marco Aliprandini

Thomas, finita la telefonata, si era sentito appesantito da qualcosa che gli si era parcheggiato, scuro e fastidioso, in un angolo dello stomaco. Da giorni, sentiva, proprio nello stomaco, quella sensazione di vuoto che il non riuscire ad esprimere i suoi sentimenti gli faceva provare. — Komisch! — aveva detto a mezzavoce, andando verso la cucina. Fuori c'era un caldo insolito e nella sua mansarda i muri sembravano sudare. Thomas amava l'inverno. Il calore secco dei termosifoni, il piumino senza lenzuola, i cappotti e le giacche di lana. Amava l'inverno perché dopo una giornata intera in ospedale, non si sentiva in colpa di aver voglia di starsene a casa. — Komisch! — aveva detto di nuovo ad alta voce, ripercorrendo la telefonata con Ada. La solita telefonata delle otto e mezza. Ogni giorno alla solita ora, le solite cose. Quasi fosse inevitabile ritrovarsi anche nell'amore nei rassicuranti confini del già detto, del già sentito per l'ennesima volta. - Sì, domani arriverò verso l'una [...] qui fa un caldo insopportabile $[\ldots]$ farò il viaggio insieme a Christine $[\ldots]$ -

Ada viveva a Milano, lui a Merano. Si erano conosciuti due anni prima ad un congresso sulla predisposizione genetica dei tumori al retto e al colon e all'inizio si erano parlati in inglese. Si erano avvicinati in una lingua tra loro equidistante. Una lingua neutra, che proprio per la sua mancanza di odore, aveva facilitato il primo approccio rendendolo apparentemente professionale.

All'inizio la comunicazione era stata formale, ma dopo alcuni minuti di inglese, Thomas e Ada si erano scoperti entrambi con passaporto italiano. Certo, la pronuncia tedesca di Thomas poteva essere fraintesa e, come al solito, lui si era quasi divertito a raccontare di essere di madrelingua e cittadinanza diversa.

Avevano mangiato insieme e Ada si era mostrata incuriosita da quello strano uomo che, spesso, parlando si fermava, quasi le parole fossero degli utensili molto delicati, da maneggiare con cura. Alle volte si era anche chiesta se questa prudenza nel parlare non fosse dovuta ad una sottile incertezza nell'uso di una lingua non propria. Una lingua che nasceva come fredda costruzione del cervello e non come calda necessità dello stomaco.

La serata era stata piacevole, lenta e leggera. Ada e Thomas il giorno dopo si erano cercati con gli occhi nell'atrio dell'albergo, che ospitava il convegno. 
Poi si erano seduti accanto e, lasciandosi andare alle improvvise accelerazioni dei sentimenti, si erano sentiti vicini, complici di qualcosa che ancora non riuscivano ad afferrare.

I bambini di madrelingua italiana disegnano il sole con le guance paffute e una pipa, che quasi si confonde con i suoi raggi. La luna come una donna misteriosa con grandi occhi indecifrabili, avvolta da una notte carica di stelle. I bambini di madrelingua tedesca invece disegnano der Mond, la luna, come un vecchio signore saggio e distante e die Sonne, il sole, come una donna radiosa con il naso piccolo e la labbra sorridenti. In apparenza, è una questione banale di articoli. Gli articoli determinativi maschili il, der diventano il disegno di un uomo e gli articoli determinativi femminili la, die quello di una donna. Solo il genere in questo caso cambia. La mamma, die Mutter o der Vater, il papà, calzano, nella stessa misura, all'esempio. Solo che in questi casi coincidono, nelle due lingue, sia i generi che gli articoli. Ogni lingua, in pratica, ha una sua struttura molecolare che la fa diventare madrelingua, lingua madre, lingua capace di generare i medesimi disegni in tutti i bambini.

Ada, finita la telefonata con Thomas, aveva acceso il televisore. Non aveva voglia di uscire e allo stesso tempo aveva voglia di non sentire silenzio intorno a sé. La giornata era stata particolarmente dura e in sala operatoria c'erano stati momenti di grande nervosismo. Il tumore, dalle pareti dello stomaco, aveva intaccato anche il peritoneo e il paziente si era riempito di liquido. Quasi sei litri.

Thomas al telefono era stato distante, di poche parole, ma per Ada questo era solo il suo modo tedesco di esprimere i sentimenti. Lo conosceva ormai da due anni e in fondo si era abituata a quella sotterranea difficoltà nel lasciarsi andare. Dapprima aveva pensato che fosse una questione di lingua. Thomas però parlava benissimo l'italiano, quindi era qualcos'altro che lo rendeva diverso. Anche quando facevano l'amore non diceva mai niente e questo ad Ada quasi piaceva perché lei aveva campo libero e lo sommergeva con il suo grande bisogno di dare voce alle cose. Forse lei era più mediterranea, più italiana, ma ripensandoci bene questo non era del tutto esatto, visto che suo padre, nato a Montebelluna, era sempre stato di poche parole.

Anche Thomas aveva acceso il televisore. Lo faceva di rado, erano pochi, infatti, i programmi che riuscivano ad interessarlo. Nel primo canale austriaco c'era una tavola rotonda dove più persone discutevano sul concetto di profugo nel XXI secolo. Dopo aver seguito alcuni interventi, Thomas si era stancato e aveva abbassato del tutto il volume. Così era rimasto a lungo davanti al video ad osservare come i vari interlocutori parlavano. Quasi per gioco aveva studiato i loro gesti, la loro mimica più o meno accentuata, poi si era chiesto se, in un mondo senza audio, sarebbe riuscito a riconoscere la nazionalità dei parlanti.

Di notte aveva fatto un sogno confuso, una vicenda senza un senso apparente, anzi un sovrapporsi frammentario di storie senza un denominatore comune. Prima che la sveglia suonasse aveva sognato di Theo, il suo gatto, e al risveglio aveva pensato che in tedesco gatto poteva essere maschile, der Kater, 
e femminile, die Katze. Aveva pensato a Theo che era un Kater tigrato e che era morto, quando lui aveva tredici anni, sotto le ruote della Mercedes di un turista germanico.

La morte, der Tod. Quando un famosissimo regista del Nord Europa in un suo film rappresentò la morte con una figura maschile, i critici dell'area mediterranea salutarono questo fatto come qualcosa di eccezionale. La morte, che ogni bambino italiano si raffigura in vesti femminili era diventata uomo, quasi scavalcando e, quindi, rinnovando l'immaginario comune. Nel Nord Europa, ad esempio in Germania, questo fatto invece era passato del tutto inosservato, anche perché ogni bambino tedesco disegna la morte come un uomo, visto che il sostantivo Tod è preceduto dall'articolo determinativo maschile der.

Se si considera che nel nostro pianeta ci sono oltre una cinquantina di lingue, cresce in proporzione, la possibilità di incontrare raffigurazioni diverse del medesimo oggetto. Raffigurazioni che possono generare una variopinta ricchezza di prospettive ma, nello stesso tempo, anche barriere scure e invalicabili. Tutto poi diventa ancora più significativo se si inizia a tener presente il fitto pulviscolo dei vari dialetti, che sono autentiche lingue madri, lingue in continuo movimento su cui poggiano le interpretazioni individuali della realtà circostante.

Antonio era arrivato a Merano con il padre che proveniva da un paese vicino a Cosenza. A casa sua, nonostante più di un quarto di secolo di Sudtirolo, parlavano ancora in dialetto, quindi lui si ritrovava a pensare utilizzando le strutture del dialetto, dovendo servire clientela sia italiana che tedesca in un locale di Gais, in Val Pusteria.

Gais è il nome di una delle poche località sudtirolesi che nessuno, nemmeno durante l'italianizzazione fascista, si sia mai preso la briga di tradurre. Anche Vierschach, piccolo paese a tre chilometri dal confine austriaco, erano riusciti a tradurlo in Versciaco e proprio a Versciaco era nata Verena, di madrelingua tedesca, ma perfettamente bilingue perché da piccola aveva sempre giocato con i figli dei finanzieri.

Antonio aveva conosciuto Verena casualmente ad una cena con amici e, dopo un breve fidanzamento, malvisto da entrambe le famiglie, si erano sposati. Avevano avuto due bambini che trascorrevano molto del loro tempo nella malga dei nonni materni. La sorella di Verena, Christine, aveva studiato medicina a Innsbruck e, dopo la laurea, era andata a specializzarsi in un ospedale di Milano, dove aveva conosciuto Ada e tramite lei anche Thomas.

Verena e sua sorella Christine a casa dei genitori parlavano dialetto pusterese. Antonio parlava calabrese e spesso si chiedeva, dopo aver discusso con Thomas, come i suoi due figli avrebbero disegnato la luna, visto che lui si rivolgeva loro in un italiano standard, quasi privo di inflessioni e la madre in dialetto.

Thomas, nel frattempo, si era alzato con la morte del suo gatto in testa e quasi per distrarsi, mentre si faceva la barba, si era messo a pensare ad Ada, che alle una sarebbe arrivata. Komisches Gefühl, come avrebbe potuto dirlo in italiano. Das Gefühl era facilmente traducibile visto che il suo unico significa- 
to è: il sentimento. Komisch si poteva tradurre con strano, non di certo con comico. Strano, però a Thomas suonava troppo impersonale per un sentimento.

Nell'aggettivo qualificativo komisch c'era una vena di leggerezza che nella sua traduzione si perdeva. C'era come un alito di vento che in strano veniva a mancare, rendendo la frase quasi irreparabile. Lui avrebbe voluto dire ad Ada che, da un po' di tempo, si sentiva distante, strano, ma non definitivamente strano, komisch appunto. Nel senso che quello che aveva lo appagava, ma non del tutto. In altre parole avrebbe voluto dirle che desiderava qualcosa di più di quel vedersi a singhiozzo tra Milano e Merano, di quelle solite telefonate, alla solita ora. Questo le avrebbe voluto dire, ma sapeva che sarebbe stato nuovamente frainteso. Sapeva che le parole spesso sono contenitori troppo definitivi per riportare l'inconsistenza dei sentimenti.

Michael, il padre di Verena e Christine, era morto due giorni prima. Una malattia lunga e dolorosa, partita dal pancreas, probabilmente causata da un eccessivo uso di bevande alcoliche. Il suo corpo si era lentamente asciugato diventando, giorno dopo giorno, come una tavola di legno ben levigata. Poi era arrivato l'ultimo respiro che, nonostante fosse da tempo previsto, aveva sorpreso i familiari. In qualche modo di fronte alla morte, maschile o femminile che sia, si rimane come sorpresi della sua esistenza.

Il funerale era stato fissato per le quindici. Antonio aveva indossato il suo vestito scuro, l'unico che possedeva e anche i bambini erano stati vestiti da grandi. Thomas, alle una, era andato a prendere alla stazione Ada e Christine $\mathrm{e}$ in macchina non avevano quasi parlato.

Molti del paese sarebbero andati al funerale di Michael, che tutti conoscevano come Much e l'anziano parroco, già dopo mangiato, aveva ordinato sul tavolo della sacrestia quello che gli sarebbe servito per la funzione.

Ogni tanto, soprappensiero, sfiorava gli oggetti con la delicatezza con cui si raddrizza un quadro alla parete dopo averlo spolverato. Avrebbe detto le solite parole di conforto e di speranza, ma questa volta, ingenuamente, gli sarebbe piaciuto dire qualcosa anche in italiano per Antonio, che spesso lo aveva aiutato nell'orto dietro alla chiesa

Anche nei funerali le usanze sono diverse. Il colore del lutto in gran parte dell'Oriente è il bianco, mentre in Occidente è il nero. Nei paesi sudtirolesi è tradizione, dopo la sepoltura, ritrovarsi in un locale a mangiare qualcosa, una minestra o dello speck, insieme ai parenti del defunto. Quasi un segnale che la vita in ogni caso deve andare avanti sugli stessi binari.

Ada, di solito sempre all'altezza della situazione, una volta entrata nel Gasthof, una specie di hotel che nei paesi ha la funzione anche di ristorante e di bar, era rimasta senza parole. Quando Christine le aveva detto di suo padre, subito, si era offerta di accompagnarla, ma adesso tra gente straniera, si sentiva inadeguata. Thomas, che parlava in dialetto con un signore molto distinto, le sembrava diverso da quello che lei conosceva. Guardandolo si era stupita di non poterlo capire. La sua gestualità era quasi più cruda come se d'improvviso si fosse intrisa di terra. Lui era un altro. Anche Christine, vicino alla 
madre, aveva un'altra faccia, parlava in un'altra maniera. E questo essere altro delle persone a lei care, dapprima l'aveva fatta quasi soffrire. Come se in qualche modo la loro diversità fosse stata un muro insuperabile o ancora peggio un tradimento.

Sulla strada verso casa, in macchina, quel sentimento era andato sciogliendosi e, come una porta che improvvisamente si apre, ad Ada parve, in quel pomeriggio, di aver in parte capito il silenzio di Thomas, o forse di aver capito qualcosa di impercettibile, ma rilevante che per due anni le era del tutto sfuggito.

Nelle lingue occidentali il pronome personale che indica la prima persona singolare io è spesso molto semplice da pronunciare. In inglese e tedesco o in francese e spagnolo è forse una delle prime parole compiute che un bambino riesce ad emettere con una certa chiarezza.

Io, che in italiano è formato solo da due vocali, in giapponese si frantuma in una serie di io femminili e maschili, formali ed informali. L'io femminile o l'io pronunciato in un contesto formale si dice watashi, quattro consonanti e tre vocali. Di sicuro watashi è un suono composto, che si riuscirà a dire in maniera chiara solo dopo un certo esercizio. L'io in giapponese si impara quindi più tardi e questo forse coincide con una sua rilevanza minore rispetto ad altre parole. Anche il colore del sole cambia. Il sole, infatti, per ogni giapponese è rosso, mentre per ogni europeo è giallo e sarebbe, probabilmente, troppo riduttivo pensare al colore della bandiera.

Thomas chiuso il libro di un anziano etnologo, vissuto per anni in Giappone, aveva spento la luce. Nel buio però non riusciva a smettere di seguire lo strano gioco che, nelle ultime settimane, lo aveva occupato quasi completamente. Così pensava che il sinonimo di buio in italiano poteva essere l'oscurità, anche se oscurità, forse per il suo articolo femminile, gli pareva rappresentare un nero meno assoluto di quello di buio. In tedesco il buio diventa neutro das Dunkel, ma il suo possibile sinonimo è anche un sostantivo femminile, die Dunkelheit.

Da giorni Thomas rifletteva sugli articoli, che precedono i nomi facendoli diventare disegni. Pensava al fatto che in giapponese non ci sono articoli e che in inglese invece non sono divisi per genere. Da qualche parte aveva letto che in Giappone i bambini disegnano il sole, Taiyoo, come una donna e la luna, Tuky, come un uomo. Poi quell'anomalia dell'articolo neutro che gli era sempre sembrato equidistante, cioè distante allo stesso modo da tutte le cose, proprio come lo scialle che sua nonna portava anche d'estate. Neutri nella sua madrelingua erano anche tutti i verbi sostantivati, das Atmen, il respirare.

Ada dormiva accanto a lui, forse da ore, così Thomas iniziò ad ascoltare, nel silenzio della stanza, il ritmo del suo respiro. Lo sentiva neutro, come neutro sentiva il silenzio quasi fossero, insieme allo scialle della nonna scomparsa alcuni anni prima, due luoghi privi di spazio e di tempo dove potersi finalmente capire. 


\section{Merano-Meran}

Per diventare adulto

ho dovuto attraversare il fiume

che divide via Enrico Toti

da quel lungo viale di tigli

dove adesso abito.

Da allora / intorno a me

non è cambiato molto!

Le bandiere - come le parole -

sono ancora esposte a giorni alterni

e delle volte penso sia

questa difficile convergenza di linguaggi

che fa di te Merano-Meran

un'anomala città

(Marco ALIPRANDRINI)

\section{Meran-Merano}

Um erwachsen zu werden

mußte ich den Fluß überqueren

der Via Enrico Totti trennt

von der langen Lindenallee

wo ich jezt wohne.

Seit damals / hat sich ringsum

nicht viel verändert

Fahnen und Wörter

immer noch ausgehängt an alternierenden Tagen

und manchmal denke ich es ist

dieser erschwerte Gleichklang der Sprachen

der aus dir Meran-Merano

eine eigenartige Stadt macht

und aus mir / deinen eigenartigen Bewohner

(Traduzione Sepp Mall) 